University of Nebraska - Lincoln

DigitalCommons@University of Nebraska - Lincoln

Faculty Publications: Materials Research

Science and Engineering Center

Materials Research Science and Engineering

Center

2007

\title{
Creating micro- and nanostructures on tubular and spherical surfaces
}

O. Lima

University of Nebraska - Lincoln

L. Tan

University of Nebraska-Lincoln, Itan4@unl.edu

A. Goel

University of Nebraska - Lincoln

Mehrdad Negahban

University of Nebraska - Lincoln, mnegahban1@unl.edu

Z. Li

Peking University, China

Follow this and additional works at: https://digitalcommons.unl.edu/mrsecfacpubs

Part of the Materials Science and Engineering Commons

Lima, O.; Tan, L.; Goel, A.; Negahban, Mehrdad; and Li, Z., "Creating micro- and nanostructures on tubular and spherical surfaces" (2007). Faculty Publications: Materials Research Science and Engineering Center. 64.

https://digitalcommons.unl.edu/mrsecfacpubs/64

This Article is brought to you for free and open access by the Materials Research Science and Engineering Center at DigitalCommons@University of Nebraska - Lincoln. It has been accepted for inclusion in Faculty Publications:

Materials Research Science and Engineering Center by an authorized administrator of DigitalCommons@University of Nebraska - Lincoln. 


\title{
Creating micro- and nanostructures on tubular and spherical surfaces
}

\author{
O. Lima, L. Tan, ${ }^{\text {a) }}$ A. Goel, and M. Negahban \\ Department of Engineering Mechanics and Nebraska Center for Materials and Nanoscience, \\ University of Nebraska, Lincoln, Nebraska 68588-0526 \\ Z. Li \\ Department of Mechanics and Engineering Science, Peking University, China, 100083
}

(Received 10 June 2007; accepted 8 October 2007; published 10 December 2007)

\begin{abstract}
The authors developed a new technique to create micro- and nanometer scale structures on curved free-standing objects by combining embossing/imprinting lithography approaches with mechanical loadings on elastic films. Embossing/imprinting generates small structures and mechanical loading determines shape or geometry of the final object. As a result, a portion of the tubes with a radius between 0.5 and $3.5 \mathrm{~mm}$ and a portion of the spheres with a radius between 2.4 and $7.0 \mathrm{~mm}$ were fabricated with grating line features (period of $700 \mathrm{~nm}$ ) and microlens array features (lens radius of $2.5 \mu \mathrm{m}$ ) atop, respectively. It was found that both static analyses and finite element models can give good estimates on the radii of those curved objects, based on the dimension of the two layers, loading format, as well as mechanic strains. Thus, good control over shape and dimension of the free-standing structure can be achieved. (C) 2007 American Vacuum Society.
\end{abstract}

[DOI: $10.1116 / 1.2804428$ ]

\section{INTRODUCTION}

Nature has been a source and inspiration to the fabrication and manufacturing community by presenting biological components with structures for incredible functions. To name a few, fibrous nanostructures, abundant on the walls of blood vessels, ${ }^{1-3}$ could regulate tensile strength of the vessel membrane and even promote cellular attachment after injury. Nanoridge features on compound eyes ${ }^{4-6}$ allows flies to have a fast response with a large field-of-view, despite the compact size of individual eyes. Fibrous muscle has regular distributions over a diaphragm, ${ }^{7}$ allowing maximum and reliable volume change during repetitive respiratory pumping. While biologically promising structures, it proves to be very challenging to generate biomimicking structures to match the criteria of their biological counterparts. One of the challenges involves creating tiny and regularly distributed structures atop curved platforms.

Patterning atop curved subjects, especially surfaces of hollow and soft tubes or spheres, challenges a good number of lithography approaches. Among viable means, embossing/ imprinting based nanolithography appears to be a good candidate due to their easy-to-process nature and structuring capability for nanometer scale features. For instance, nonplanar lithography techniques developed in embossing community ${ }^{8-11}$ have advantages by directly building nanostructures on final objects. An efficient material transfer is a prerequisite to allow the formation of those nanostructures during the contact between the rigid curvature with a soft and patterned surface. Seemingly easy processes, they require precise handling of the curved surfaces during alignment steps. And potential limitation on types of materials exists due to the transferring nature of those processes. An

a) Author to whom correspondence should be addressed; electronic mail: ltan4@unl.edu alternative approach demonstrated by Lee ${ }^{6}$ extends the fabrication capability to soft spherical objects, where an embossing technique is integrated with a mechanical deformation process. In their approach, nanostructures were first imprinted atop a planar and elastic membrane; then, the medium was deformed by a negative pressure in a sealed environment. Compatibility with a variety of lithographic processes and benefits of good accuracy in alignment imply great advantages. Design complexity, however, might be associated with their process when a free-standing object is demanded or the target object has a tubular or irregular shape or geometry. A key gap exists to develop an embossing/ imprinting based patterning technique for free-standing soft objects of variety curvatures, such as tubular [onedimensional (1D)], spherical [two-dimensional (2D)], or more complex structures; and at the same time, delivering good accuracy in alignment steps or having compatibility with conventional planar lithography platforms.

This acute need forms the scope of this article. We report our efforts, an integration of embossing with mechanical deformation on layered elastic films, to address this need by demonstrating tubular and spherical structures with micro- or nanometer features on surfaces. Moreover, it was found that both static analyses and finite element models can give us good estimates on the shape of those curved objects after fabrication. Thus, control over the shape and dimension of the soft objects can be achieved by tuning the layered structure, loading format, as well as mechanic strains. Simplicity of our approach suggests great potential to mimicking functionalities in biological counterparts in the future.

\section{EXPERIMENT}

Poly(dimethylsiloxane) (PDMS) (Sylgard 184®, Dow Corning) was used to fabricate curved and free-standing structures in our proposed nanofabrication processes. The 
(a)

Step 1

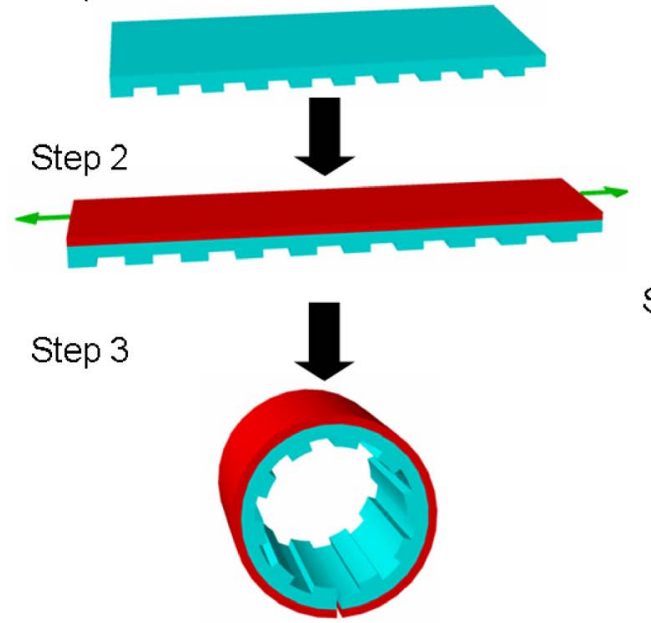

Step 4

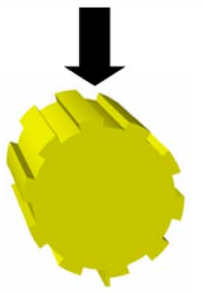

New, Fresh Layer

Substrate. Previously stretched layer.
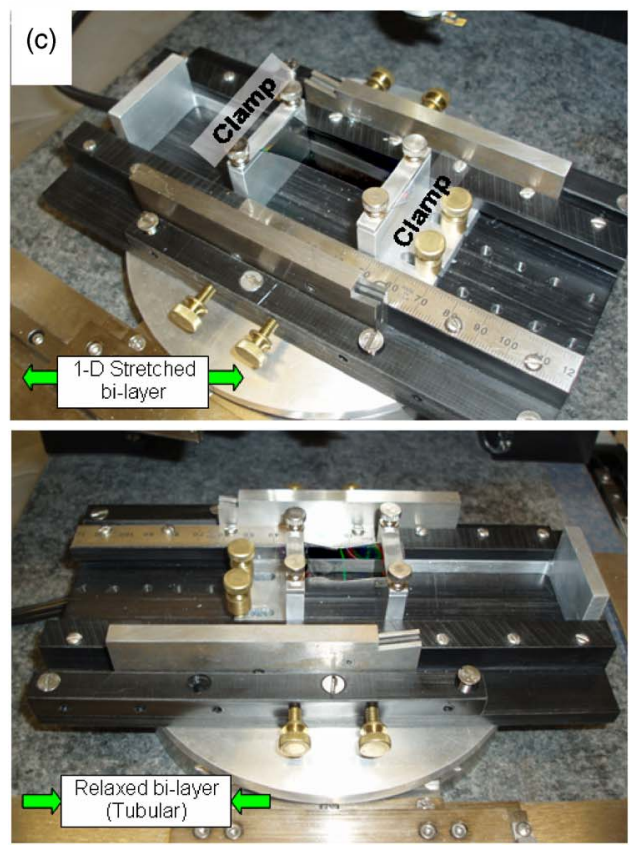

(b)

Step 1
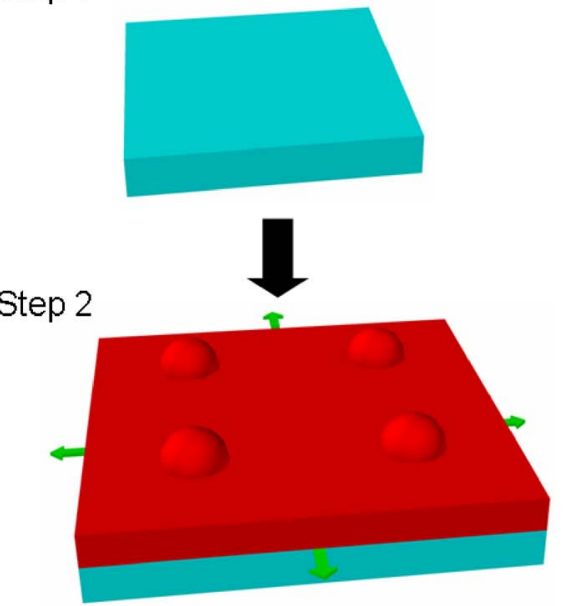

Step 3
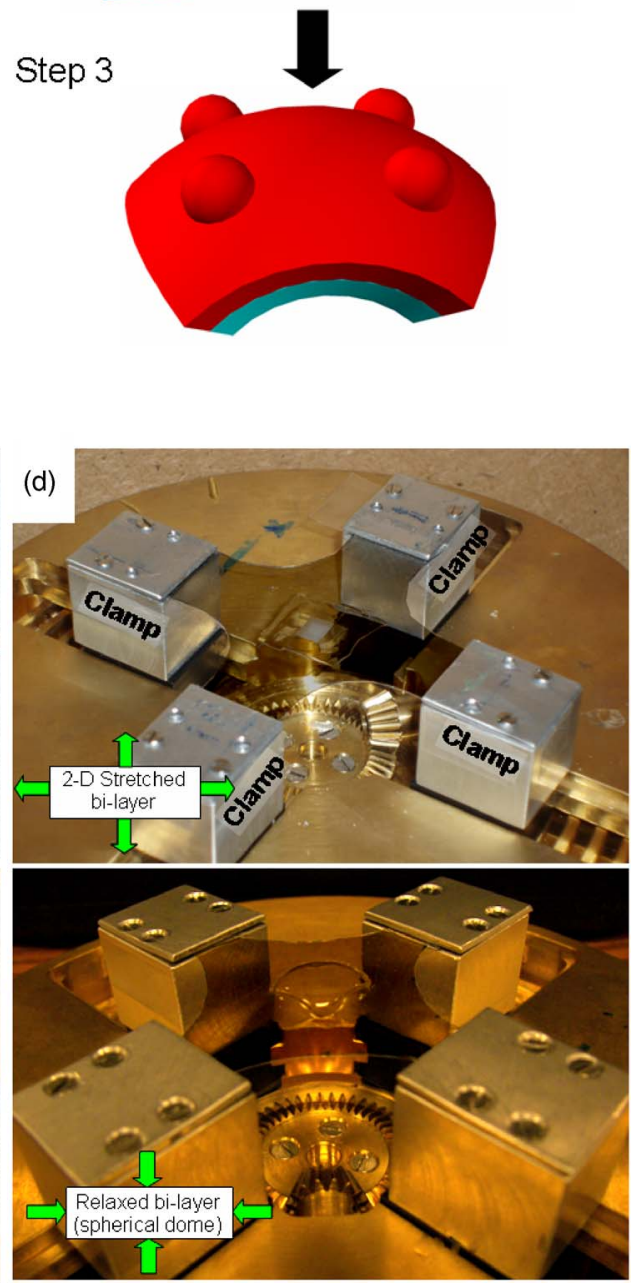

FIG. 1. (a) Schematic process flow to produce nanostructures on cylindrical objects: (Step 1) embossing/imprinting patterned soft film with features on the bottom; (Step 2) stretch the film in 1D fashion and cast a liquid elastomer precursor, then bake to form a bilayer structure; (Step 3) relax the bilayer to form a portion of tube; and (Step 4) replicate the free-standing portion of the tube into a portion of a rigid cylinder. (b) Process flow to produce nanostructures on spherical objects: (Step 1) prepare a soft film as substrate; (Step 2) stretch the film in 2D fashion and embossing/imprinting a new layer with patterns;and (Step 3) relax the bilayer to form a portion of the sphere. Homemade stretching tools for (c) 1D and (d) 2D manipulation. Elastic film can be fixed between solid bars indicated in (c) and (d). Translation of these bars introduces 1D or 2D deformation to the soft films in between. elastic films were prepared first by vigorous mixing of a 10:1 ratio of part $\mathrm{A}(5.0 \mathrm{~g})$ and part $\mathrm{B}(0.5 \mathrm{~g})$ in a plastic beaker followed by degassing in a vacuum chamber. Subsequently, the mixture was spin coated at $300 \mathrm{rpm}$ for $30 \mathrm{~s}$ atop either a flat surface or a rigid mold. Finally, the whole assembly was baked at $80{ }^{\circ} \mathrm{C}$ for $2 \mathrm{~h}$ before the solid PDMS was released.

Two rigid molds with different surface features were used. The first mold is composed of silicon and has a grating line feature (a linewidth of $350 \mathrm{~nm}$, a pitch size of $700 \mathrm{~nm}$, and a depth of $120 \mathrm{~nm}$ ); the second one is composed of thermoplastic polymer (polystyrene) and has a close-packed honeycomb feature (recessed half-spheres with a diameter of $2.5 \mu \mathrm{m})^{12}$

Homemade stretchers, allowing either 1D or 2D mechanical manipulation, were designed to facilitate our fabrication processes. The stretcher provides (see details in Sec. III) ap- 
proximate uni- or biaxial deformation to PDMS films and generates objects with desired shape or geometries. It is worthy to note that the film was cut with an aspect ratio (length:width) of 5:1, which makes the narrowing effect at the center during manipulation negligible. Similarly, our finite element analysis (FEA) simulations modeled the deformation in the central area of the cross-finger shaped film only, where the deformation can be regarded as biaxial. Our optical measurements of the curvature were performed through a ML8000 Meiji microscope or a JEOL 840A scanning electron microscope (SEM). SEM was also used to image nanostructures atop curved surfaces. UV exposure was performed with a Dymax light curing system (model 5000) and the irradiation intensity was measured by a UVP-UVX radiometer integrated with UVX-36 sensor, with an attenuator ratio of 10:1.

\section{RESULTS AND DISCUSSION}

\section{A. Nanostructures on tubular surfaces}

Approximations to portions of tubes were fabricated following the process flow shown in Fig. 1(a). In this process, patterned PDMS thin film was first mounted on a 1D stretcher [Fig. 1(c)], with nanostructures facing down [step 1, Fig. 1(a)]. The nanostructures were fabricated following conventional embossing/imprinting lithography, where grating lines with a pitch of $700 \mathrm{~nm}$ and a linewidth of $350 \mathrm{~nm}$ were obtained. The stretcher is then allowed to deform the film until reaching a desired strain; a fresh PDMS precursor is cast atop the stretched film and baked to form a bilayer membrane structure [step 2, Fig. 1(a)]. During the baking process, the PDMS precursor could penetrate into the stretched solid film and this will give rise to a tight bonding between the two layers. After the stretcher is released, the strain in the bottom PDMS layer was relaxed, which compresses the top PDMS layer and generate a free-standing and soft portion of tube [step 3, Fig. 1(a)]. Finally, epoxy was used to fill the soft portion of the tube and revealed to us a portion of a rigid cylinder, with surface nanostructures copied from the inner side of the soft portion of the tube [step 4, Fig. 1(a)]. Figure 2 presents both optical and SEM micrographs of the final free-standing portion of the tube [inset-left, Figs. 2(a) and 2(b)], rigid replica [inset-right, Figs. 2(a) and 2(b)], and surface nanostructures [Figs. 2(a) and 2(b)]. These two rigid replicas have radius of 0.5 and $3.5 \mathrm{~mm}$, respectively. SEM images in Fig. 2 show the nanostructures on surfaces of rigid replica. These tiny structures are negative copies of the gratings inside the free-standing object, with a linewidth of 350 $\mathrm{nm}$ and a pitch dimension of $700 \mathrm{~nm}$. It is worthwhile to note that variation of the image contrast from top to bottom in Fig. 2(b) indicates the inward bending of the rigid replica with respect to the paper.

Differences in the aforementioned radius are caused by the applied initial strains, i.e., $40 \%$ or $80 \%$. A larger strain introduces a more significant compression to the top PDMS layer, resulting in a much smaller radius in the final tubular structure. A static model can be constructed to provide a
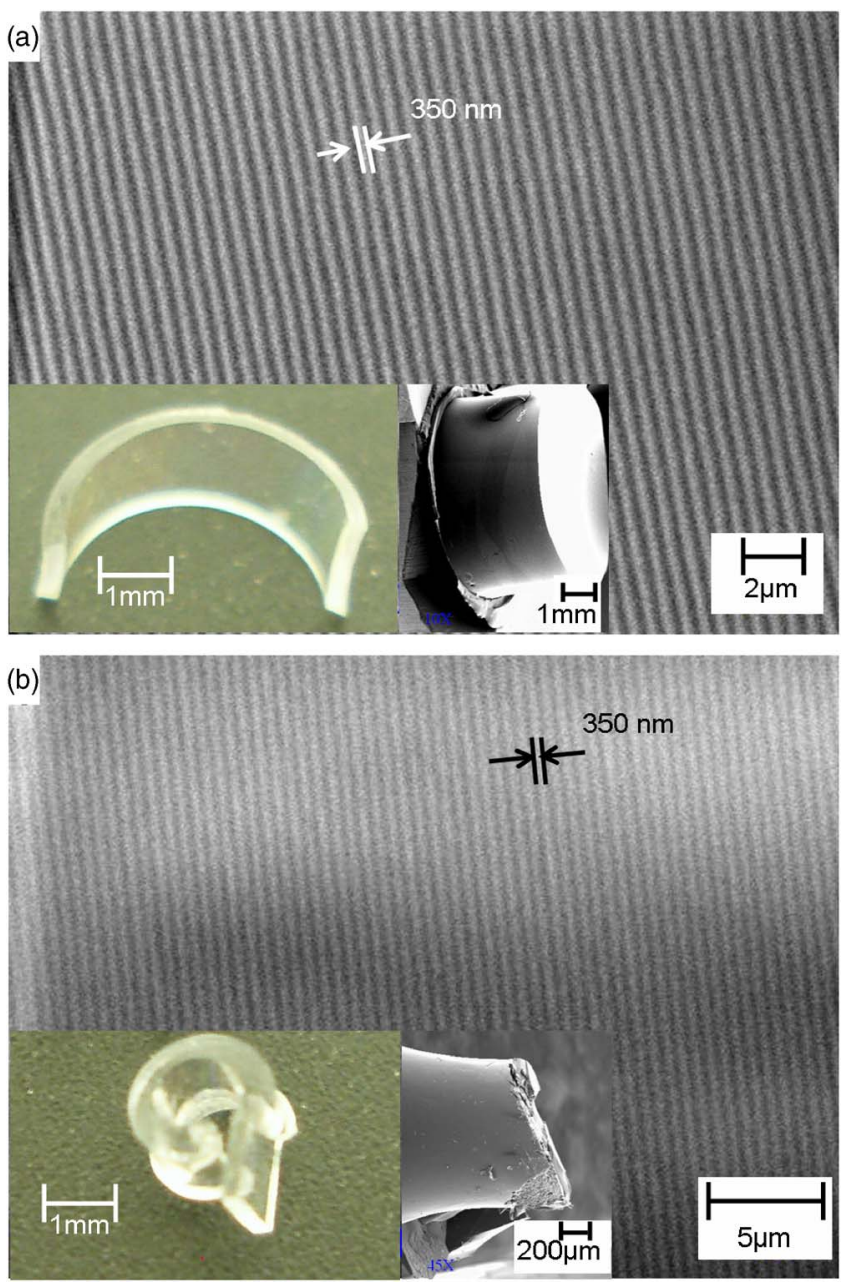

FIG. 2. SEM images of grating line features fabricated atop curved surface of portions of rigid cylinders. Insets: free-standing bilayer portions of tubes (left) and rigid replica (right). The free-standing tubular structures were received by applying uniaxial strain at $40 \%$ [(a) inset] and $80 \%$ [(b) inset], respectively.

qualitative analysis for the tubular formation as shown in Fig. 3. In this model, we assume only linear and elastic deformation occurring in the film. The film has an initial shape and geometry as shown in Fig. 3(a). After an external strain of $\varepsilon_{0}$ is applied, the film becomes longer and thinner, with different dimensions shown in Fig. 3(b). When a fresh PDMS with the same shape and geometry as the stretched ones is used as a boundary layer [Fig. 3(b)], much limited relaxation will occur along the interface between both PDMS layers; and a compressive stress will be associated to the top membrane, with the interface experiencing a maximum compression. Therefore, a tubular structure with an internal radius of $r$ and a bending angle of $\theta$ will form in this relaxed bilayer structure as shown in Fig. 3(c). From this free-body diagram and using classic static theory, ${ }^{13}$ we can assume a neutral plane is located in a $\rho$ distance away from the tubular center and the longitudinal dimension of this plane should have the same value as $l_{f}$ or $\theta \rho$. That is to say, at a location of $y$ distance away from this neutral plane, longitudinal dimension follows Eq. (1): 
(a)

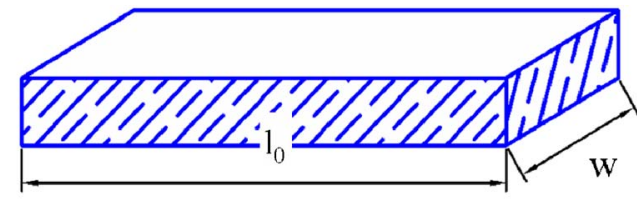

(b)

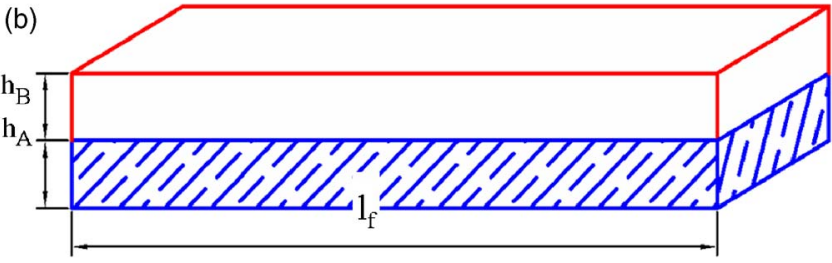

(c)

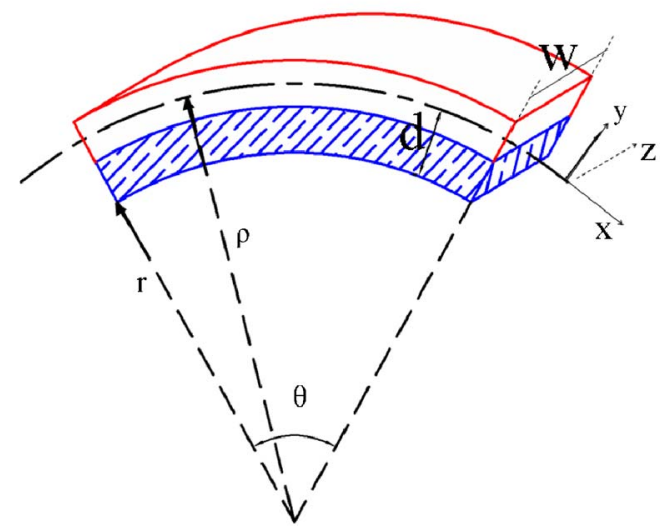

FIG. 3. Static model for predicting the radius of curvature of the tubular structure: (a) unstretched PDMS film; (b) stretched film A with additional layer B cast atop; and (c) free body diagram of the relaxed bilayer structure.

$$
l=\theta(\rho+y) \text { and } l_{0}=\theta \rho /\left(1+\varepsilon_{0}\right) .
$$

Accordingly, the internal strains in both PDMS layers can be described as

$$
\begin{aligned}
& \varepsilon_{A}=\frac{l-l_{0}}{l_{0}}=\frac{\theta(\rho+y)-\theta \rho\left(\frac{1}{1+\varepsilon_{0}}\right)}{\theta \rho\left(\frac{1}{1+\varepsilon_{0}}\right)}=\frac{y+y \varepsilon_{0}+\rho \varepsilon_{0}}{\rho}, \\
& \varepsilon_{B}=\frac{l-l_{f}}{l_{f}}=\frac{\theta(\rho+y)-\theta \rho}{\theta \rho}=\frac{y}{\rho} .
\end{aligned}
$$

Under static equilibrium, the integration of normal stresses generated over the transversal $y z$ plane [Fig. 3(c)] is zero

$$
\begin{aligned}
& \int_{A} \sigma_{A} d A+\int_{B} \sigma_{B} d A=0 \text { or } \\
& \int_{A} E_{A} \frac{y+y \varepsilon_{0}+\rho \varepsilon_{0}}{\rho} w d y+\int_{B} E_{B} \frac{y}{\rho} w d y=0,
\end{aligned}
$$

where $E_{A}$ and $E_{B}$ represent the elastic modulus of both layers. Equation (4) can be expanded to be

$$
\frac{E_{A} w}{\rho} \int_{-d}^{-d+h_{A}}\left[y\left(1+\varepsilon_{0}\right)+\rho \varepsilon_{0}\right] d y+\frac{E_{B} w}{\rho} \int_{-d+h_{A}}^{-d+h_{A}+h_{B}} y d y=0
$$

where $d$ is the distance from the bottom of the PDMS layer, i.e., $\rho-r$, to the neutral plane.

Likely, integration of the moment over the neutral axis of $z$ is also zero under a static equilibrium

$$
\begin{aligned}
& \int_{A} \sigma_{A} y d A+\int_{B} \sigma_{B} y d A=0 \text { or } \\
& \int_{A} E_{A} \frac{y+y \varepsilon_{0}+\rho \varepsilon_{0}}{\rho} y w d y+\int_{B} E_{B} \frac{y}{\rho} y w d y=0,
\end{aligned}
$$

which can be expanded as

$$
\frac{E_{A} w}{\rho} \int_{-d}^{-d+h_{A}}\left[y^{2}\left(1+\varepsilon_{0}\right)+\rho \varepsilon_{0} y\right] d y+\frac{E_{B} w}{\rho} \int_{-d+h_{A}}^{-d+h_{A}+h_{B}} y^{2} d y=0 .
$$

Integrations in both Eqs. (5) and (7) gave us the tubular radius as shown in Eq. (8),

$$
r=\frac{\left(k_{E}^{2} k_{h}^{4}+4 k_{E} k_{h}^{3}+6 k_{E} k_{h}^{2}-3 k_{E} k_{h}^{2} \varepsilon_{0}-2 k_{E} k_{h} \varepsilon_{0}+4 k_{E} k_{h}+\varepsilon_{0}+1\right) h_{A}}{6 k_{E} k_{h} \varepsilon_{0}\left(1+k_{h}\right)},
$$

where $k_{E}=E_{B} / E_{A}$ and $k_{h}=h_{B} / h_{A}$.

Above, static analysis is carried out with the assumption that only linear and small deformation occurred in the process. Therefore, the elastic modulus in both layers is equal, i.e., $E_{B}=E_{A}$ or $k_{E}=1$. When a large or nonlinear deformation occurs in the bilayer structure, we can still apply the above methodology to the analysis by plugging a nonequal modulus into Eq. (8) or $k_{E}$. A set of bilayer structures varying on both thickness ratio $\left(h_{B} / h_{A}=10 \% \rightarrow 85 \%\right)$ and initial strain $\left(\varepsilon_{0}=10 \% \rightarrow 110 \%\right)$ were prepared to verify this treatment. It was found that good fitting on experimental data can be achieved with value of $k_{E}$ fluctuating between 0.1 and 0.5 [Fig. 4(a)]. This can be interpreted to mean that the modulus in our stretched PDMS is larger than that in a compressed mode. ${ }^{14,15}$ And this modulus variation can be as large as tenfold. Even though such a huge modulus change is possible for elastic materials, possible plastic flow might occur under such a condition and this awaits further investigation. While qualitative, our analysis did suggest that the dimension of the layered structure $\left(k_{h}\right)$, external strain $\left(\varepsilon_{0}\right)$, and material properties $\left(k_{E}\right)$ are important measures to govern the final shape of the bilayer structure. It is worthwhile to note that the earlier loading/unloading process [Fig. 1(a)] could also be used as a miniaturization technique to regulate pattern density and dimension. ${ }^{16,17}$ In this case, a thin layer, instead of a thick PDMS, is favored as a top layer for a dramatic dimension change. Accordingly, a small value of $k_{h}$ will give rise to a relatively flat surface which is favored for uniformity control in conventional miniaturizations.

To further evaluate our experimental data, we conducted a nonlinear and large deformation FEA. Symmetry of the load- 

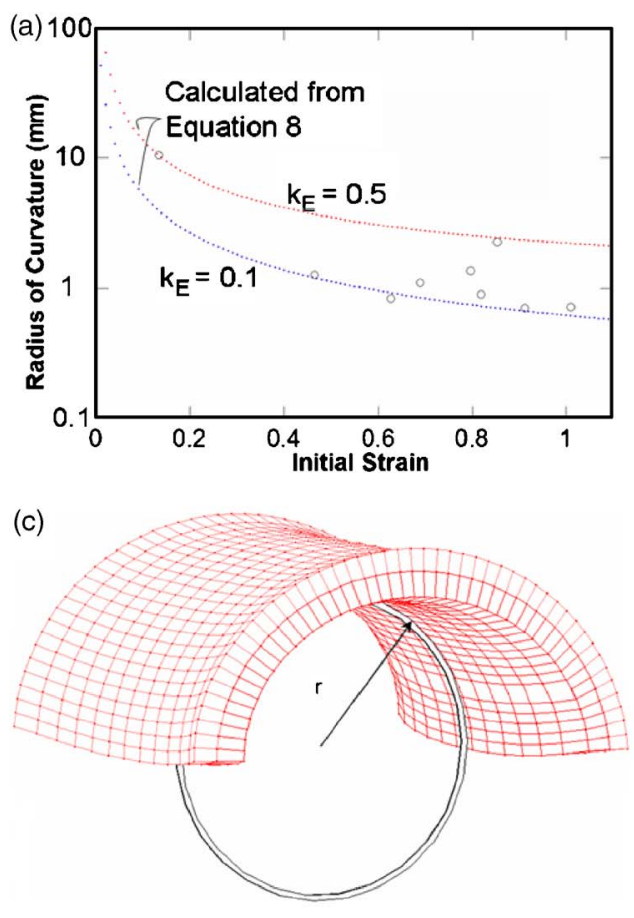
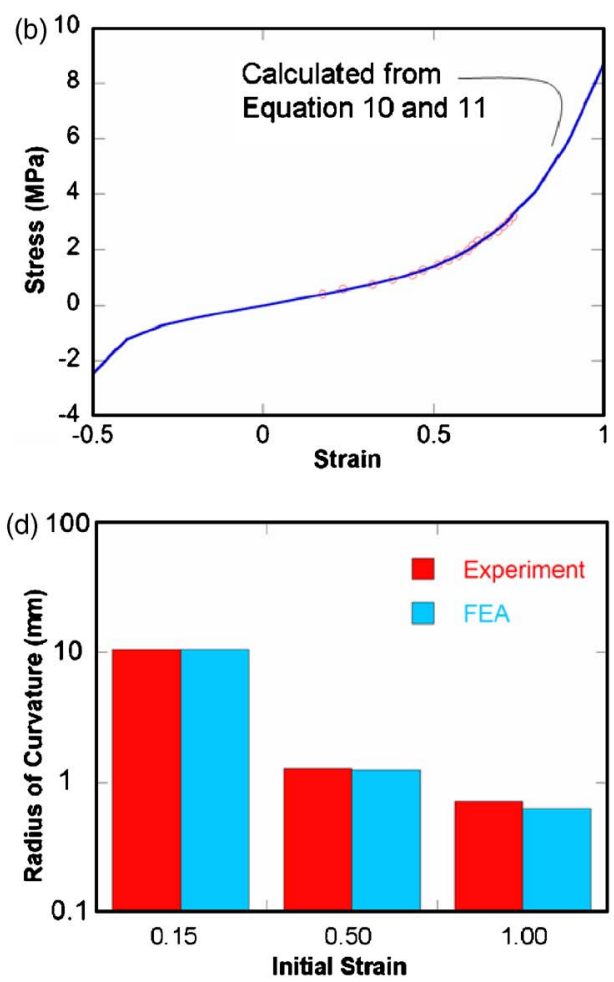

FIG. 4. (a) Plot of the tubular radius calculated from a static analysis and experiments with respect to the initial strain applied. $k_{E}$ is the ratio of elastic modulus between top and bottom layer; (b) stress-strain curve for a tension test performed on PDMS and corresponding fitting with a material model; (c) FEA mesh for the relaxed bilayer structure, and radius measurement is performed at the center to avoid the edge effect; and (d) comparison between FEA predicted radius and experimental data under three different strains. ing condition allowed us to consider one fourth of the bilayer structure in $x z$ plane with boundary conditions. Prestretched and unstretched 27-noded brick elements are used for the bottom and top layer, respectively. FEMSolid, programmed by two of the authors (A.G. and M.N.), has the capability to support our nonlinear and large deformation analyses.

In modeling the elastic response of the material, it is assumed the material is initially isotropic at the time of formation and characterized by a specific free-energy $\psi$ that is a function of the isotropic invariants of the left Cauchy stretch tensor $\mathbf{B}=\mathbf{F}^{T} \mathbf{F}$, where $\mathbf{F}$ is the deformation gradient. In particular it is assumed that $\psi$ depends on the two invariants $I_{1}^{*}$ and $J$, where $J=\operatorname{det}(\mathbf{F})$ is the volume ratio and $I_{1}^{*}$ $=\operatorname{tr}(\mathbf{B}) / J^{2 / 3}=I_{1} / J^{2 / 3}$. For a thermoelastic material, the Cauchy stress $\mathbf{T}$ can be derived from the free energy from the relation

$$
\mathbf{T}=\rho \partial_{\mathbf{F}}(W) \mathbf{F}^{T},
$$

where $\rho$ denotes density. Letting $G=2 \rho_{0}\left(\partial \psi / \partial I_{1}^{*}\right)$ and assuming quadratic dependence of free-energy with volumetric strain results in the expression for stress given by

$$
\mathbf{T}=\kappa(J-1) \mathbf{I}+G J^{-5 / 3}\left[\mathbf{B}-\frac{\operatorname{tr}(\mathbf{B})}{3} \mathbf{I}\right]
$$

In the analysis, the bulk modulus $\kappa$ was taken large to simulate incompressible material response. The modulus $G$ was fit to the experimental stress $\sim$ strain data, assuming the extension to occur without volume change. The resulting fit of $G$ is given by the expression

$$
\begin{aligned}
G(P a)= & 31.3 \times 10^{3}\left(I_{1}^{*}-3\right)^{2}-47.5 \times 10^{3}\left(I_{1}^{*}-3\right) \\
& +25.7 \times 10^{4} .
\end{aligned}
$$

Finally, Eqs. (10) and (11) are used as the material model for the simulation. As can be seen from Fig. 4(b), a good fitting with experimental data is received. Furthermore, a total of three strain conditions, i.e., $\varepsilon_{0}=15 \%, 50 \%$, and $100 \%$, are used for rest of the FEA modeling. Representative FEA mesh is shown in Fig. 4(c), along with a circle to indicate the associated radius measurement. Figure 4(d) indicates a good fitting between experimentally measured radii and calculated ones from those FEA. This further verifies our earlier statement on the tubular shape of the final bilayer structure.

\section{B. Nanostructure on spherical surfaces}

Approximations to portions of spheres other than cylindrical ones can be created by modifying the loading conditions in Fig. 1(b). Particularly, a flat film of PDMS, instead of patterned ones in 1D loading, is mounted on a $2 \mathrm{D}$ stretcher [step 1, Fig. 1(b)]. The stretcher is then allowed to deform the film until a desired strain; a fresh PDMS precursor is cast, embossed, and baked atop the stretched film [step 2, Fig. 1(b)]. After the stretcher is released, a free-standing and soft portion of sphere structure is formed [step 3, Fig. 1(b)].

The SEM image in Fig. 5(a) shows the close-packing features on the rigid mold. This mold was used to emboss a PDMS bilayer copy [step 2, Fig. 1(b)]. Subsequently, this sample is baked to cure the liquid PDMS and soaked in toluene to remove the mold material, that is, polystyrene. After drying, stress release in step 3 leads to a final structure of spherical shape with microlens array patterns on surface [Fig. 5(b)]. Figure 5(c) shows a zoom-in image of the close- 

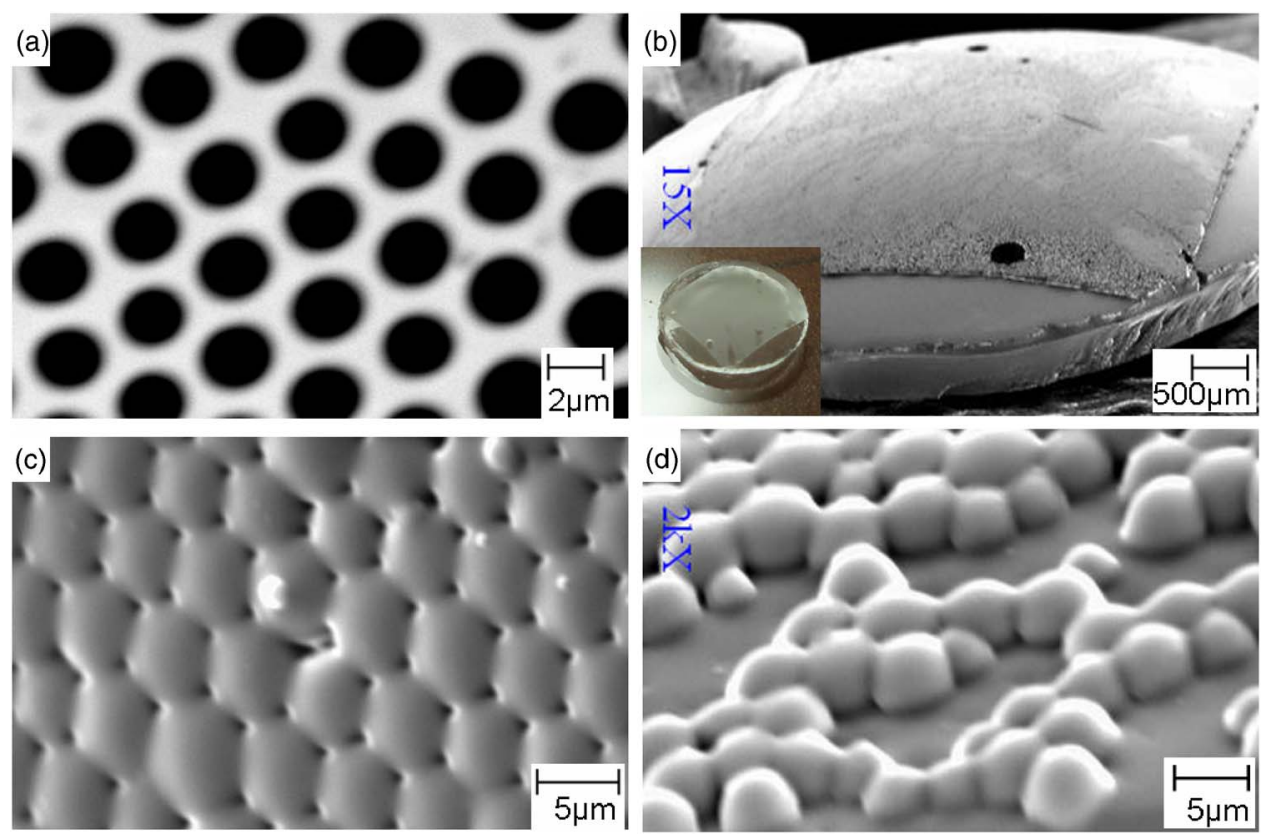

FIG. 5. SEM images of (a) polystyrene mold with close-packed features; (b) the portion of sphere structure with microlens array; (c) top view of the zoom-in image at the center of the portion of sphere, where the close-packed arrays can be seen; and (d) sideview along the edge of the portion of sphere, where a loosely packed location was picked to show the profile of individual structures for microlenses. packed features on this curved surface, where the radius is $7.0 \mathrm{~mm}$ and each individual lens has a radius of $2.5 \mu \mathrm{m}$ [Fig. 6(d)].

To confirm control over the radius of curvature, a bilayer PDMS structure was prepared and the bottom layer was applied with a biaxial strain of $31 \%$. After releasing the loading, a portion of sphere was formed with a radius of $2.4 \mathrm{~mm}$. FEA was used again to model one fourth of the layer, which has prestretched and unstretched 24 noded brick-type elements for the bottom and top layer, respectively. The radius

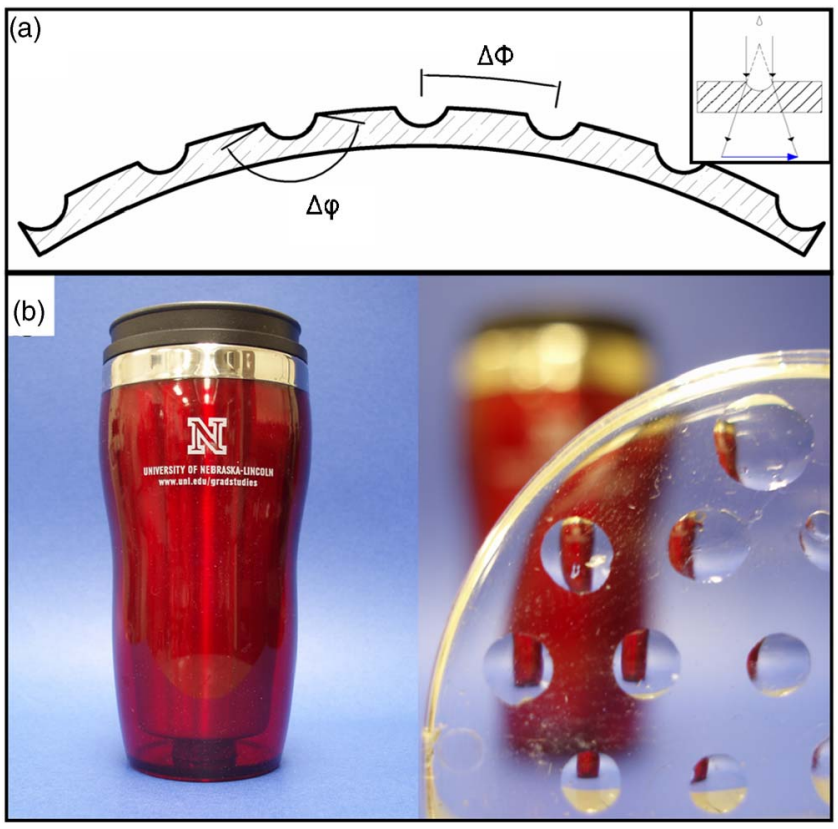

FIG. 6. (a) Illustration of a multilens imaging system with concave lenses. $\Delta \varphi$ and $\Delta \Phi$ are parameters related to the increment of FOV and (b) reduced images produced by an array of lens, each one focused on different locations of the object. calculated from FEA is $2.7 \mathrm{~mm}$ and, once again this confirms that control over curvature is possible by tuning both the layer structure and the amount of strain applied to the substrate.

Additionally, our structure can be used to mimic a compound eye. Figure 6(a) illustrates the operation of this multilens imaging system with convex lens array atop a curved surface. Field-of-view (FOV) for each lens on curved surface depends on viewing angle parameters, such as $\Delta \phi$ and $\Delta \varphi{ }^{18}$ Such a mechanism is evidenced by the smaller images seen in Fig. 6(b). Furthermore, we let UV irradiation to pass through a flat PDMS, as well as those macroscopic convex lenses. The UV intensity measured dropped from 60.0 for the flat surface to $40.0 \mathrm{~mW} / \mathrm{cm}^{2}$ for the convex lens. This light intensity diminish further confirms the spreading effect of the convex lens as indicated in Fig. 6(a).

\section{CONCLUSIONS}

We demonstrated a novel approach to obtain free-standing curved objects with nanostructures on surfaces. Embossing/ imprinting was used to generate small structures and mechanical loading determines the shape or geometry of the final object. As a result, a portion of the tubes with a radius between 0.5 and $3.5 \mathrm{~mm}$ and a portion of the spheres with a radius between 2.0 and $7.0 \mathrm{~mm}$ were fabricated with grating line features (period of $700 \mathrm{~nm}$ ) and microlens array features (lens radius of $2.5 \mu \mathrm{m}$ ) atop, respectively. It was found that both static analyses and finite element models can give us good estimates on radii of those curved objects based on the layered structure, loading format, as well as mechanic strains. Advantages of this new approach lie on yielding freestanding soft objects, regulating shapes and dimensions, and have good compatibilities with conventional lithography 
platforms. Therefore, this technique could have impact in the general lithography community and is suitable for application in biology, optics, and mechanics.

\section{ACKNOWLEDGMENTS}

The project described was made possible by Nebraska Center for Energy Science and Research and NSF MRSEC. One of the authors, Z.L., was partially supported by the exchange program between Peking University and the University of Nebraska-Lincoln and O.L. appreciates many helpful discussions with Jie Xu.

\footnotetext{
${ }^{1}$ L. Buttafoco, P. J. Dijkstra, A. A. Poot, I. Vermes, and J. Feijen, J. Controlled Release 87, 295 (2003).

${ }^{2}$ P. Buijtenhuijs et al., Biotechnol. Appl. Biochem. 39, 141 (2004).

${ }^{3}$ T. Takei, S. Sakai, T. Yokonuma, H. Ijima, and K. Kawakami, Biotechnol. Prog. 23, 182 (2007).

${ }^{4}$ G. A. Horridge, H. C. Loughuethiggins, and G. A. Horridge, Philos, Trans. R. Soc. London, Ser. B 337, 271 (1992).

${ }^{5}$ J. Duparre, P. Dannberg, P. Schreiber, A. Brauer, and A. Tunnermann,
}

Appl. Opt. 44, 2949 (2005).

${ }^{6}$ K. H. Jeong, J. Kim, and L. P. Lee, Science 312, 557 (2006).

${ }^{7}$ P. Gamba, M. T. Conconi, R. Lo Piccolo, G. Zara, R. Spinazzi, and P. P. Parnigotto, Pediatr. Surg. Int. 18, 327 (2002).

${ }^{8}$ J. A. Rogers, R. J. Jackman, G. M. Whitesides, J. L. Wagener, and A. M. Vengsarkar, Appl. Phys. Lett. 70, 7 (1997).

${ }^{9}$ X. D. Huang, L. R. Bao, X. Cheng, L. J. Guo, S. W. Pang, and A. F. Yee, J. Vac. Sci. Technol. B 20, 2872 (2002)

${ }^{10}$ L. Tan, Y. P. Kong, L. R. Bao, X. D. Huang, L. J. Guo, S. W. Pang, and A. F. Yee, J. Vac. Sci. Technol. B 21, 2742 (2003).

${ }^{11}$ W. R. Childs and R. G. Nuzzo, Adv. Mater. 16, 1323 (2004).

${ }^{12}$ M. Srinivasarao, D. Collings, A. Philips, and S. Patel, Science 292, 79 (2001).

${ }^{13}$ J. M. Gere, Mechanics of Materials, 5th ed. (Broks/Cole, Pacific Grove, CA, 2001).

${ }^{14}$ K. K. Liu, D. R. Williams, and B. J. Briscoe, J. Phys. D 31, 294 (1998).

${ }^{15}$ H. Schmid and B. Michel, Macromolecules 33, 3042 (2000).

${ }^{16}$ L. Tan, Z. Ouyang, M. Liu, J. Ell, J. Hu, T. E. Patten, and G. Y. Liu, J. Phys. Chem. B 110, 23315 (2006).

${ }^{17}$ Z. Q. Ouyang, L. Tan, M. Z. Liu, O. S. Judge, X. D. Zhang, H. Li, J. Hu, T. E. Patten, and G. Y. Liu, Small 2, 884 (2006).

${ }^{18}$ J. Duparre, P. Dannberg, P. Schreiber, A. Brauer, and A. Tunnermann, Appl. Opt. 43, 4303 (2004). 